Appl. Set-Valued Anal. Optim. 3 (2021), No. 3, pp. 293-308

Available online at http://asvao.biemdas.com

https://doi.org/10.23952/asvao.3.2021.3.04

\title{
ON CONVEXITY AND QUASICONVEXITY OF EXTREMAL VALUE FUNCTIONS IN SET OPTIMIZATION
}

\author{
T. GERLACH* S. ROCKTÄSCHEL
}

Institute of Mathematics, Technische Universität Ilmenau, Po 1005 65, D-98684 Ilmenau, Germany

\begin{abstract}
We study different classes of convex and quasiconvex set-valued maps defined by means of the $\ell$-less relation and the $u$-less relation. The aim of this paper is to formulate necessary and especially sufficient conditions for the convexity/quasiconvexity of extremal value functions.
\end{abstract}

Keywords. Convex functions; Extremal functions; Quasiconvex functions; Set optimization.

\section{INTRODUCTION}

The classical concepts of convexity and quasiconvexity of real-valued functions have been extended to set-valued maps in various ways (see e.g. [1]). In doing so the fundamental importance of these concepts in optimization theory is carried over to set-valued optimization. Thereby, most of the extensions are based on set relations induced by a convex cone $C$. These relations allow to compare sets as a whole and provide a solution concept for set optimization problems, the so-called set approach. In this paper two of them, the $\ell$-less relation and the $u$-less relation (see $[2,3,4]$ ), will be studied.

In [5], it was shown (see the forthcoming Theorem 2.1) that comparing sets with respect to these relations is (under additional assumptions) equivalent to comparing the function values of special extremal functions defined by using all elements $l$ of the dual cone $C^{\star}$. This is a fundamental result for set-valued optimization, since it allows to consider an equivalent vectorvalued optimization problem instead of a given set-valued optimization problem. Thereby, the components of the objective funtion of the vector-valued optimization problem are the minimum value functions or the maximum value functions for the respective elements $l$ of the dual cone $C^{\star}$. This is the reason why it is very interesting to examine how certain properties can be guaranteed for these extremal functions. In particular, we are interested in conditions that, imposed on the objective function of a set-valued optimization problem, are sufficient for the convexity and the quasiconvexity of all the minimum value functions or all the maximum value functions mentioned above. In this respect, it is well known that for the $\ell$-less relation there is an equivalent characterization of $\ell$-type $C$-convex set-valued maps by using the convexity of all the minimum value functions (see [6] and the forthcoming Theorem 3.1). As far as we know, there

\footnotetext{
*Corresponding author.

E-mail addresses: tobias.gerlach@tu-ilmenau.de (T. Gerlach), stefan.rocktaeschel@tu-ilmenau.de (S. Rocktäsc hel).

Received January 13, 2021; Accepted July 28, 2021.
}

(C)2021 Applied Set-Valued Analysis and Optimization 
is no similar result with an equivalent characterization for $u$-type $C$-convex set-valued maps by using the convexity of all coresponding maximum value functions in the existing literature. We will formulate and proof such a result in the forthcoming Theorem 3.2.

Moreover, a systematization and an in-depth analysis of various quasiconvexity concepts for set-valued maps which in most cases generalize the $\ell$-type $C$-convexity or the $u$-type $C$ convexity are presented in [7]. Note that neither for the $\ell$-less relation nor for $u$-less relation there exists a known concept for quasiconvexity of set-valued maps such that the quasiconvexity of a set-valued map is equivalent to the fact that the corresponding extremal value functions are quasiconvex for all $l \in C^{\star}$. Using the hierarchical order of the various quasiconvexity concepts for set-valued maps given in [7] we will formulate and proof with Theorem 4.1 and Theorem 4.2 at least partial answers to the questions which quasiconvexity concepts are in both cases sufficient to guarantee the quasiconvexity of all the corresponding extremal value functions.

In Section 2 we state the basic definitions and conventions which we need in the following. The convexity of set-valued maps and their extremal value functions is the topic of the following Section 3. Finally, Section 4 is devoted to the various quasiconvexity concepts for set-valued maps and its impact on the quasiconvexity of the corresponding extremal value functions. In connection to this, we consider the quasiconvexity concepts based on the $\ell$-less relation in Subsection 4.1 and the quasiconvexity concepts based on the $\ell$-less relation in Subsection 4.2.

\section{PRELIMINARIES}

Throughout the paper we use the notation $\overline{\mathbb{R}}$ for the extended real numbers defined by $\overline{\mathbb{R}}:=$ $\mathbb{R} \cup\{-\infty, \infty\}$ and use the following conventions

$$
\inf \emptyset:=+\infty, \sup \emptyset:=-\infty \text {, and }(+\infty)+(-\infty)=(-\infty)+(+\infty)=+\infty .
$$

Based on this, a convex extended real-valued function is defined as follows (see for instance [6]):

Definition 2.1. Let $S$ be a nonempty convex subset of a real linear space $X$. Then an extended real-valued function $\varphi: S \rightarrow \overline{\mathbb{R}}$ is called convex on $S$, if for all $x^{1}, x^{2} \in S$ and all $\lambda \in(0,1)$ it holds

$$
\varphi\left(\lambda x^{1}+(1-\lambda) x^{2}\right) \leq \lambda \varphi\left(x^{1}\right)+(1-\lambda) \varphi\left(x^{2}\right) .
$$

Note that by using $\lambda \in(0,1)$ instead of as usual $\lambda \in[0,1]$ the indeterminate forms $0 \cdot(+\infty)$ and $0 \cdot(-\infty)$ are avoided. The following known result provides an equivalent characterization of a convex extended real-valued function (see also for instance [6]).

Lemma 2.1. Let $S$ be a nonempty convex subset of a real linear space $X$ and let $\varphi: S \rightarrow \overline{\mathbb{R}}$ be an extended real-valued function. Then $\varphi$ is convex on $S$ if and only if its strict epigraph over $S$ defined by

$$
\operatorname{epi}_{<}(\varphi, S):=\{(x, \alpha) \in S \times \mathbb{R} \mid \varphi(x)<\alpha\}
$$

is a convex set.

The following remark clarifies the importance of the last convention of (2.1).

Remark 2.1. Note that the third convention of (2.1) is indispensable with regard to Definition 2.1 and Lemma 2.1. To see this we consider the extended real-valued function $\varphi$ defined 
by

$$
\varphi: S=\mathbb{R} \rightarrow \overline{\mathbb{R}} \text { with } \varphi(x):= \begin{cases}-\infty, & \text { if } x \in(-\infty, 0) \\ +\infty, & \text { if } x \in[0,+\infty)\end{cases}
$$

Then the strict epigraph over $S=\mathbb{R}$ is given by

$$
\operatorname{epi}_{<}(\varphi, S)=\{(x, \alpha) \in \mathbb{R} \times \mathbb{R} \mid \varphi(x)<\alpha\}=\{(x, \alpha) \in \mathbb{R} \times \mathbb{R} \mid x \in(-\infty, 0), \alpha \in \mathbb{R}\}
$$

and is obviously convex. According to Lemma 2.1 this is equivalent to the fact that the function $\varphi$ is convex. Let now $x^{1}:=-x$ and $x^{2}:=x$ with $x \in(0,+\infty)$. Then we obtain by the definition of $\varphi$, by the choice of $x^{1}$ and $x^{2}$, and by the convexity of $\varphi$ according to Definition 2.1

$$
+\infty=\varphi(0)=\varphi\left(\frac{1}{2} x^{1}+\frac{1}{2} x^{2}\right) \leq \frac{1}{2} \varphi\left(x^{1}\right)+\frac{1}{2} \varphi\left(x^{2}\right)=\frac{1}{2}(-\infty)+\frac{1}{2}(+\infty)=(-\infty)+(+\infty),
$$

which can only be fulfilled if $(-\infty)+(+\infty)=+\infty$ holds. Any other convention would lead to a contradiction here.

Besides convexity properties of functions and maps we also consider several concepts of quasiconvexity in this paper. Therefore, we recall the definition of quasiconvex extended realvalued functions (see again for instance [6]).

Definition 2.2. Let $S$ be a nonempty convex subset of a real linear space $X$. Then an extended real-valued function $\varphi: S \rightarrow \overline{\mathbb{R}}$ is called quasiconvex on $S$, if for all $x^{1}, x^{2} \in S$ and all $\lambda \in[0,1]$ it holds

$$
\varphi\left(\lambda x^{1}+(1-\lambda) x^{2}\right) \leq \max \left\{\varphi\left(x^{1}\right), \varphi\left(x^{2}\right)\right\} .
$$

In analogy to Lemma 2.1 there exists also a known equivalent characterization of a quasiconvex extended real-valued function (see also for instance [6]).

Lemma 2.2. Let $S$ be a nonempty convex subset of a real linear space $X$ and let $\varphi: S \rightarrow \overline{\mathbb{R}}$ be an extended real-valued function. Then $\varphi$ is quasiconvex on $S$ if and only if its strict level set over $S$ defined by

$$
\varphi_{<}^{-1}(S, \alpha):=\{x \in S \mid \varphi(x)<\alpha\}
$$

is a convex set for all $\alpha \in \mathbb{R}$.

Moreover, an extended real-valued function $\varphi: S \rightarrow \overline{\mathbb{R}}$ is obviously quasiconvex on $S$ if and only if $x^{1}, x^{2} \in S$ with $\varphi\left(x^{1}\right) \leq \varphi\left(x^{2}\right)$ implies that $\varphi\left(\lambda x^{2}+(1-\lambda) x^{1}\right) \leq \varphi\left(x^{2}\right)$ for all $\lambda \in[0,1]$.

For two subsets $S$ and $T$ of a real linear space we denote by $S+T:=\{s+t \mid s \in S, t \in T\}$ and by $S-T:=\{s-t \mid s \in S, t \in T\}$ the algebraic sum and the algebraic difference of $S$ and $T$, respectively. Moreover, for $S$ and an arbitrary $\alpha \in \mathbb{R}$ we use the notation $\alpha S:=\{\alpha s \mid s \in S\}$.

A nonempty subset $C$ of a real linear space $Y$ is called a cone if $y \in C$ and $\lambda \geq 0$ imply $\lambda y \in C$. A cone $C$ is convex if and only if $C+C=C$ holds. Moreover, a convex cone $C \subset Y$ induces a partial ordering of $Y$ defined by

$$
y^{1} \leq_{C} y^{2} \Leftrightarrow y^{2}-y^{1} \in C
$$

for all $y^{1}, y^{2} \in Y$.

A vector-valued map $f: S \subset X \rightarrow Y$, where $X$ and $Y$ are real linear spaces, $Y$ is partially ordered by a convex cone $C \subset Y$, and $S$ is a nonempty convex subset of $X$, is called

- $C$-convex on $S$ if, for all $x^{1}, x^{2} \in S$ and all $\lambda \in(0,1)$, it holds $f\left(\lambda x^{1}+(1-\lambda) x^{2}\right) \leq C$ $\lambda f\left(x^{1}\right)+(1-\lambda) f\left(x^{2}\right)$ (cf. [8, Definition 2.4]) and 
- quasiconvex (in the sense of Jahn) on $S$ if $x^{1}, x^{2} \in S$ with $f\left(x^{1}\right) \leq_{C} f\left(x^{2}\right)$ implies that $f\left(\lambda x^{1}+(1-\lambda) x^{2}\right) \leq_{C} f\left(x^{2}\right)$ for all $\lambda \in[0,1]$ (cf. [8, Definition 7.9]).

Note that in the special case $Y=\mathbb{R}$ and $C=\mathbb{R}_{+}=[0, \infty)$ the definition of a $\mathbb{R}_{+}$-convex and of a quasiconvex (in the sense of Jahn) vector-valued map coincides with the (usual) definition of a convex and of a quasiconvex real-valued function $\varphi: S \rightarrow \mathbb{R}$, respectively.

Furthermore, if a real linear space $Y$ is partially ordered by a convex cone $C \subset Y$, then $C$ also induces the two binary relations $\preccurlyeq_{C}^{\ell}$ (called the $\ell$-less relation) and $\preccurlyeq_{C}^{u}$ (called the $u$-less relation) on the power set of $Y$. These were introduced by Kuroiwa in [2, 3, 4] and are defined by

$$
A \preccurlyeq_{C}^{\ell} B: \Leftrightarrow B \subset A+C \text { and } A \preccurlyeq_{C}^{u} B: \Leftrightarrow A \subset B-C
$$

for $A, B \subset Y$. Note that by using $\emptyset \pm C=\emptyset$ it holds

$$
A \preccurlyeq_{C}^{\ell} \emptyset \text { and } \emptyset \preccurlyeq_{C}^{u} A \text { for all } A \subset Y,
$$

and it holds

$$
\emptyset \npreceq_{C}^{\ell} A \text { and } A \aleph_{C}^{u} \emptyset \text { for all } A \subset Y \text { with } A \neq \emptyset \text {. }
$$

We use the notations $\aleph_{C}^{\ell}$ and $\aleph_{C}^{u}$ to indicate that the considered relations $\preccurlyeq_{C}^{\ell}$ and $\preccurlyeq_{C}^{u}$ are not satisfied. If $Y$ is additionally a real topological linear space, then we denote by $Y^{\star}$ the topological dual space of $Y$ and by $C^{\star}:=\left\{l \in Y^{\star} \mid l(k) \geq 0 \forall k \in C\right\}$ the dual cone of $C$. Theorem 2.1 follows by the proofs of [5, Lemma 2.1, Theorem 2.1] and by [5, Remark 2.1]. It provides an important characterization of the set relations $\preccurlyeq_{C}^{\ell}$ and $\preccurlyeq_{C}^{u}$.

Theorem 2.1. [5] Let $A$ and $B$ be subsets of a real locally convex space $Y$ partially ordered by a convex cone $C \subset Y$. Then it holds:

(i)

$$
\begin{array}{ll}
A \preccurlyeq_{C}^{\ell} B \Rightarrow \forall l \in C^{\star} \backslash\left\{0_{Y^{\star}}\right\}: & \inf _{a \in A} l(a) \leq \inf _{b \in B} l(b) \text { and } \\
A \preccurlyeq_{C}^{u} B \Rightarrow \forall l \in C^{\star} \backslash\left\{0_{Y^{\star}}\right\}: & \sup _{a \in A} l(a) \leq \sup _{b \in B} l(b) .
\end{array}
$$

(ii) If the set $A+C$ is closed and convex, then

$$
A \preccurlyeq_{C}^{\ell} B \Leftrightarrow \forall l \in C^{\star} \backslash\left\{0_{Y^{\star}}\right\}: \inf _{a \in A} l(a) \leq \inf _{b \in B} l(b) .
$$

If the set $B-C$ is closed and convex, then

$$
A \preccurlyeq_{C}^{u} B \Leftrightarrow \forall l \in C^{\star} \backslash\left\{0_{Y^{\star}}\right\}: \sup _{a \in A} l(a) \leq \sup _{b \in B} l(b) .
$$

\section{CONVEXITY OF SET-VALUED MAPS AND EXTREMAL VALUE FUNCTIONS}

As usual in set-valued analysis, for a given set-valued map $F: S \rightrightarrows Y$, where $X$ and $Y$ are real linear spaces and $S$ is a nonempty convex subset of $X$, we define its domain by

$$
\operatorname{dom}(F, S):=\{x \in S \mid F(x) \neq \emptyset\} .
$$

The following convexity concepts for set-valued maps have been introduced in [9].

Definition 3.1. Let $X$ and $Y$ be real linear spaces, let $Y$ be partially ordered by a convex cone $C \subset Y$, let $S$ be a nonempty convex subset of $X$, and let $F: S \rightrightarrows Y$ be a set-valued map with $\operatorname{dom}(F, S) \neq \emptyset$. The map $F$ is called 
(a) $\ell$-type $C$-convex on $S$, if for all $x^{1}, x^{2} \in \operatorname{dom}(F, S)$ and all $\lambda \in(0,1)$ it holds

$$
F\left(\lambda x^{1}+(1-\lambda) x^{2}\right) \preccurlyeq_{C}^{\ell} \lambda F\left(x^{1}\right)+(1-\lambda) F\left(x^{2}\right) .
$$

(b) $u$-type $C$-convex on $S$, if for all $x^{1}, x^{2} \in \operatorname{dom}(F, S)$ and all $\lambda \in(0,1)$ it holds

$$
F\left(\lambda x^{1}+(1-\lambda) x^{2}\right) \preccurlyeq_{C}^{u} \lambda F\left(x^{1}\right)+(1-\lambda) F\left(x^{2}\right) .
$$

Based on this and according to [7] we denote by $\mathscr{C}_{\ell}(S, Y, C)$ and $\mathscr{C}_{u}(S, Y, C)$ the classes of all $\ell$ type $C$-convex set-valued maps on $S$ and all $u$-type $C$-convex set-valued maps on $S$, respectively. Note that the $\ell$-type $C$-convexity of a set-valued map $F$ on $S$ implies the convexity of $\operatorname{dom}(F, S)$ (cf. (2.3)) and that $F \in \mathscr{C}_{\ell}(S, Y, C)$ is equivalent to the fact that the epigraph of $F$ defined by $\operatorname{epi}(F, S, C):=\{(x, y) \in \operatorname{dom}(F, S) \times Y \mid y \in F(x)+C\}$ is a convex set (see for instance [8, Lemma 14.8]). In contrast to this, the $u$-type $C$-convexity of a set-valued map $F$ on $S$ does not necessarily imply the convexity of $\operatorname{dom}(F, S)$ (cf. (2.2)).

The convexity concepts for set-valued maps defined above in Definition 3.1 can be interpreted as a generalization of the $C$-convexity of vector-valued maps. Indeed, if $f: S \rightarrow Y$ is a vectorvalued map and we define the set-valued map $F: S \rightrightarrows Y$ by $F(x):=\{f(x)\}$ for all $x \in S$, i.e., it holds $\operatorname{dom}(F, S)=S$, then $f$ is $C$-convex on $S$ if and only if $F \in \mathscr{C}_{\ell}(S, Y, C)$, and $F \in \mathscr{C}_{\ell}(S, Y, C)$ if and only if $F \in \mathscr{C}_{u}(S, Y, C)$.

The following known Theorem 3.1 provides (under the assumptions of Definition 3.1) an equivalent characterization of an $\ell$-type $C$-convex set-valued map $F$ by using for $l \in Y^{\star}$ convexity properties of the composite set-valued maps $l \circ F$ defined by

$$
l \circ F: S \rightrightarrows \mathbb{R} \text { with } l \circ F(x)=l(F(x)):=\left\{\begin{array}{cl}
\bigcup_{y \in F(x)}\{l(y)\}, & \text { if } x \in \operatorname{dom}(F, S) \\
\emptyset, & \text { if } x \in S \backslash \operatorname{dom}(F, S)
\end{array}\right.
$$

and of the minimum value functions $\varphi_{\min }^{F, S, l}$ defined by

$$
\varphi_{\min }^{F, S, l}: S \rightarrow \overline{\mathbb{R}} \text { with } \varphi_{\min }^{F, S, l}(x):=\inf l \circ F(x)=\inf _{y \in F(x)} l(y) .
$$

Theorem 3.1. [6, Theorem 2.1] Let $X$ be a real linear space, let $Y$ be a locally convex space partially ordered by a convex cone $C \subset Y$, let $S$ be a nonempty convex subset of $X$, and let $F: S \rightrightarrows Y$ be a set-valued map with $\operatorname{dom}(F, S) \neq \emptyset$. If $F(x)+C$ is closed and convex for all $x \in \operatorname{dom}(F, S)$, then the following statements (i), (ii), and (iii) are equivalent:

(i) $F \in \mathscr{C}_{\ell}(S, Y, C)$.

(ii) $l \circ F \in \mathscr{C}_{\ell}\left(S, \mathbb{R}, \mathbb{R}_{+}\right)$for all $l \in C^{\star}$.

(iii) The minimum value function $\varphi_{\min }^{F, S, l}$ is convex on $S$ for all $l \in C^{\star}$.

As far as we know, there is no similar result to Theorem 3.1 with an equivalent characterization for $u$-type $C$-convex set-valued maps in the existing literature. We want to formulate such a result in the forthcoming Theorem 3.2. Therefore, in analogy to the minimum value function $\varphi_{\min }^{F, S, l}$ for $l \in Y^{\star}$ we define the maximum value function $\varphi_{\max }^{F, S, l}$ by

$$
\varphi_{\max }^{F, S, l}: S \rightarrow \overline{\mathbb{R}} \text { with } \varphi_{\max }^{F, S, l}(x):=\sup l \circ F(x)=\sup _{y \in F(x)} l(y) .
$$

In order to prove this theorem we follow roughly the approach of the proof of Theorem 3.1 in [6] and need the following three auxiliary results. In the light of this, the validity of Lemma 3.1 is obvious. 
Lemma 3.1. Let $Y$ be a real topological linear space and let $l \in Y^{\star}$. Then it holds

$$
\sup _{y \in A+B} l(y)=\sup _{a \in A} l(a)+\sup _{b \in B} l(b)
$$

for all nonempty subsets $A$ and $B$ of $Y$.

Lemma 3.2. Let $X$ be a real linear space, let $Y$ be a real topological linear space partially ordered by a convex cone $C \subset Y$, let $S$ be a nonempty convex subset of $X$, and let $F: S \rightrightarrows Y$ be a set-valued map with $\operatorname{dom}(F, S) \neq \emptyset$. If $F \in \mathscr{C}_{u}(S, Y, C)$, then it holds $l \circ F \in \mathscr{C}_{u}\left(S, \mathbb{R}, \mathbb{R}_{+}\right)$for all $l \in C^{\star}$.

Proof. Let $F \in \mathscr{C}_{u}(S, Y, C), l \in C^{\star}$, and $H:=l \circ F$. Then it follows $\operatorname{dom}(F, S)=\operatorname{dom}(H, S)$ by definition. Moreover, let $x^{1}, x^{2} \in \operatorname{dom}(F, S)=\operatorname{dom}(H, S)$ and $\lambda \in(0,1)$. We have to show that

$$
H\left(\lambda x^{1}+(1-\lambda) x^{2}\right) \subset \lambda H\left(x^{1}\right)+(1-\lambda) H\left(x^{2}\right)-\mathbb{R}_{+} .
$$

If $\lambda x^{1}+(1-\lambda) x^{2} \notin \operatorname{dom}(F, S)=\operatorname{dom}(H, S)$, then (3.1) is trivially fulfilled.

Otherwise, if $\lambda x^{1}+(1-\lambda) x^{2} \in \operatorname{dom}(F, S)=\operatorname{dom}(H, S)$, then let

$$
\bar{\alpha} \in H\left(\lambda x^{1}+(1-\lambda) x^{2}\right) .
$$

Hence, there must exist $\bar{y} \in F\left(\lambda x^{1}+(1-\lambda) x^{2}\right)$ such that

$$
\bar{\alpha}=l(\bar{y}) .
$$

Since $F \in \mathscr{C}_{u}(S, Y, C)$, it holds

$$
\bar{y} \in F\left(\lambda x^{1}+(1-\lambda) x^{2}\right) \subset \lambda F\left(x^{1}\right)+(1-\lambda) F\left(x^{2}\right)-C,
$$

and there must exist $y^{1} \in F\left(x^{1}\right), y^{2} \in F\left(x^{2}\right)$, and $k \in C$ such that

$$
\bar{y}=\lambda y^{1}+(1-\lambda) y^{2}-k \text {. }
$$

Since $l \in C^{\star}$, it holds

$$
\bar{\alpha}=l(\bar{y})=l\left(\lambda y^{1}+(1-\lambda) y^{2}-k\right)=\lambda l\left(y^{1}\right)+(1-\lambda) l\left(y^{2}\right)-l(k) \leq \lambda l\left(y^{1}\right)+(1-\lambda) l\left(y^{2}\right)
$$

and we obtain

$$
\begin{aligned}
\bar{\alpha} & \in\left\{\lambda l\left(y^{1}\right)+(1-\lambda) l\left(y^{2}\right)\right\}-\mathbb{R}_{+} \\
& \subset \lambda l\left(F\left(x^{1}\right)\right)+(1-\lambda) l\left(F\left(x^{2}\right)\right)-\mathbb{R}_{+} \\
& =\lambda H\left(x^{1}\right)+(1-\lambda) H\left(x^{2}\right)-\mathbb{R}_{+},
\end{aligned}
$$

which proves (3.1).

Lemma 3.3. Let $X$ be a real linear space, let $Y$ be a real topological linear space partially ordered by a convex cone $C \subset Y$, let $S$ be a nonempty convex subset of $X$, let $F: S \rightrightarrows Y$ be a set-valued map with $\operatorname{dom}(F, S)=S$, and let $l \in C^{\star}$. If $l \circ F \in \mathscr{C}_{u}\left(S, \mathbb{R}, \mathbb{R}_{+}\right)$, then the maximum value function $\varphi_{\max }^{F, S}$ is convex on $S$.

Proof. Let $l \in C^{\star}, l \circ F \in \mathscr{C}_{u}\left(S, \mathbb{R}, \mathbb{R}_{+}\right)$, and $H:=l \circ F$. In order to prove that the corresponding maximum value function $\varphi_{\text {max }}^{F, S}$ is convex on $S=\operatorname{dom}(F, S)=\operatorname{dom}(H, S)$, it suffices by Lemma 2.1 to show that its strict epigraph

$$
\operatorname{epi}_{<}\left(\varphi_{\max }^{F, S, l}\right):=\left\{(x, \alpha) \in S \times \mathbb{R} \mid \varphi_{\max }^{F, S, l}(x)<\alpha\right\}
$$

is a convex set.

If $\operatorname{epi}_{<}\left(\varphi_{\max }^{F, S, l}\right)=\emptyset$, then it holds $\varphi_{\max }^{F, S, l}(x)=+\infty$ for all $x \in S=\operatorname{dom}(F, S)=\operatorname{dom}(H, S)$ and 
nothing is to prove. Hence, we may assume in the following that epi $i_{<}\left(\varphi_{\max }^{F, S, l}\right) \neq \emptyset$. Let $\left(x^{1}, \alpha_{1}\right),\left(x^{2}, \alpha_{2}\right) \in \mathrm{epi}_{<}\left(\varphi_{\max }^{F, S, l}, S\right)$, i.e., it holds $-\infty<\varphi_{\max }^{F, S, l}\left(x^{1}\right)<\alpha_{1}<\infty$ and $-\infty<\varphi_{\max }^{F, S, l}\left(x^{2}\right)<$ $\alpha_{2}<\infty$, and let $\lambda \in(0,1)$. By the convexity of $S$, we obtain

$$
\lambda x^{1}+(1-\lambda) x^{2} \in S=\operatorname{dom}(F, S)=\operatorname{dom}(H, S),
$$

and we choose some $\beta_{1}, \beta_{2} \in \mathbb{R}$ such that

$$
\varphi_{\max }^{F, S, l}\left(x^{1}\right)<\beta_{1}<\alpha_{1} \text { and } \varphi_{\max }^{F, S, l}\left(x^{2}\right)<\beta_{2}<\alpha_{2} .
$$

Then, it holds

$$
H\left(x^{1}\right) \subset\left\{\beta_{1}\right\}-\mathbb{R}_{+} \text {and } H\left(x^{2}\right) \subset\left\{\beta_{2}\right\}-\mathbb{R}_{+} .
$$

Since $H \in \mathscr{C}_{u}\left(S, \mathbb{R}, \mathbb{R}_{+}\right)$, we obtain

$$
H\left(\lambda x^{1}+(1-\lambda) x^{2}\right) \subset \lambda H\left(x^{1}\right)+(1-\lambda) H\left(x^{2}\right)-\mathbb{R}_{+} \subset\left\{\lambda \beta_{1}+(1-\lambda) \beta_{2}\right\}-\mathbb{R}_{+},
$$

and thus

$$
\varphi_{\max }^{F, S, l}\left(\lambda x^{1}+(1-\lambda) x^{2}\right)=\sup H\left(\lambda x^{1}+(1-\lambda) x^{2}\right) \leq \lambda \beta_{1}+(1-\lambda) \beta_{2}<\lambda \alpha_{1}+(1-\lambda) \alpha_{2},
$$

i.e.,

$$
\lambda\left(x^{1}, \alpha_{1}\right)+(1-\lambda)\left(x^{2}, \alpha_{2}\right)=\left(\lambda x^{1}+(1-\lambda) x^{2}, \lambda \alpha_{1}+(1-\lambda) \alpha_{2}\right) \in \operatorname{epi}_{<}\left(\varphi_{\max }^{F, S, l}, S\right),
$$

which proves the convexity of epi ${ }_{<}\left(\varphi_{\max }^{F, S, l}, S\right)$.

Using the auxiliary results, we can prove the following equivalent characterization of $u$-type $C$-convex set-valued maps similar to Theorem 3.1.

Theorem 3.2. Let $X$ be a real linear space, let $Y$ be a locally convex space partially ordered by a convex cone $C \subset Y$, let $S$ be a nonempty convex subset of $X$, and let $F: S \rightrightarrows Y$ be a setvalued map with $\operatorname{dom}(F, S)=S$. If $F(x)-C$ is closed and convex for all $x \in \operatorname{dom}(F, S)$, then the following statements (i), (ii), and (iii) are equivalent:

(i) $F \in \mathscr{C}_{u}(S, Y, C)$;

(ii) $l \circ F \in \mathscr{C}_{u}\left(S, \mathbb{R}, \mathbb{R}_{+}\right)$for all $l \in C^{\star}$;

(iii) The maximum value function $\varphi_{\max }^{F, S, l}$ is convex on $S$ for all $l \in C^{\star}$.

Proof. Using Lemma 3.2 and Lemma 3.3 it remains to show that (iii) implies (i). Let therefore the maximum value function $\varphi_{\max }^{F, S, l}$ be convex on $S=\operatorname{dom}(F, S)$ for all $l \in C^{\star}$. Assume that $F \notin \mathscr{C}_{u}(S, Y, C)$, then there exist $x^{1}, x^{2} \in S=\operatorname{dom}(F, S)$ and $\lambda \in(0,1)$ such that

$$
\begin{aligned}
& F\left(\lambda x^{1}+(1-\lambda) x^{2}\right) \npreceq_{C}^{u} \lambda F\left(x^{1}\right)+(1-\lambda) F\left(x^{2}\right) \\
\Leftrightarrow & F\left(\lambda x^{1}+(1-\lambda) x^{2}\right) \not \subset \lambda F\left(x^{1}\right)+(1-\lambda) F\left(x^{2}\right)-C .
\end{aligned}
$$

By the convexity of $S=\operatorname{dom}(F, S)$, we obtain $\lambda x^{1}+(1-\lambda) x^{2} \in S=\operatorname{dom}(F, S)$. Thus

$$
F\left(\lambda x^{1}+(1-\lambda) x^{2}\right) \neq \emptyset .
$$

Hence, by Theorem 2.1 (ii), there must exist $\tilde{l} \in C^{\star} \backslash\left\{0_{Y^{\star}}\right\}$ with

$$
\varphi_{\max }^{F, S, \tilde{l}}\left(\lambda x^{1}+(1-\lambda) x^{2}\right)=\sup _{y \in F\left(\lambda x^{1}+(1-\lambda) x^{2}\right)} \tilde{l}(y)>\sup _{y \in \lambda F\left(x^{1}\right)+(1-\lambda) F\left(x^{2}\right)} \tilde{l}(y),
$$


and we obtain by Lemma 3.1 that

$$
\begin{aligned}
\varphi_{\max }^{F, S, \tilde{l}}\left(\lambda x^{1}+(1-\lambda) x^{2}\right) & >\sup _{y \in \lambda F\left(x^{1}\right)+(1-\lambda) F\left(x^{2}\right)} \tilde{l}(y) \\
& =\sup _{y^{1} \in \lambda F\left(x^{1}\right)} \tilde{l}\left(y^{1}\right)+\sup _{y^{2} \in(1-\lambda) F\left(x^{2}\right)} \tilde{l}\left(y^{2}\right) \\
& =\sup _{y^{1} \in F\left(x^{1}\right)} \tilde{l}\left(\lambda y^{1}\right)+\sup _{y^{2} \in F\left(x^{2}\right)} \tilde{l}\left((1-\lambda) y^{2}\right) \\
& =\lambda \sup _{y^{1} \in F\left(x^{1}\right)} \tilde{l}\left(y^{1}\right)+(1-\lambda) \sup _{y^{2} \in F\left(x^{2}\right)} \tilde{l}\left(y^{2}\right) \\
& \lambda \varphi_{\max }^{F, S, \tilde{l}}\left(x^{1}\right)+(1-\lambda) \varphi_{\max }^{F, S, \tilde{l}}\left(x^{2}\right),
\end{aligned}
$$

which contradicts that $\varphi_{\max }^{F, S, l}$ is convex on $S=\operatorname{dom}(F, S)$ for all $l \in C^{\star}$.

The following example shows that for the validity of Theorem 3.2 the additional assumption that $\operatorname{dom}(F, S)=S$ cannot be replaced by the weaker condition that $\operatorname{dom}(F, S)$ is a convex set.

Example 3.1. Let $X=\mathbb{R}, Y=\mathbb{R}^{2}, C=C^{\star}=\mathbb{R}_{+}^{2}, S=[-1,1]$, and the set-valued map $F: S \rightrightarrows Y$ be defined by

$$
F(x):=\left\{\begin{array}{ll}
\emptyset & , \text { if } x \in[-1,0) \\
{[1-x, 1] \times[1-x, 1]} & , \text { if } x \in[0,1]
\end{array} .\right.
$$

Then, for $l=\left(l_{1}, l_{2}\right)^{\top} \in C^{\star}=\mathbb{R}_{+}^{2}$, it holds for the composite set-valued maps $l \circ F: S \rightrightarrows \mathbb{R}$

$$
l \circ F(x)=\left\{\begin{array}{ll}
\emptyset & \text {, if } x \in[-1,0) \\
{\left[l_{1}+l_{2}-\left(l_{1}+l_{2}\right) x, l_{1}+l_{2}\right]} & , \text { if } x \in[0,1]
\end{array},\right.
$$

for the minimum value functions $\varphi_{\min }^{F, S, l}: S \rightarrow \overline{\mathbb{R}}$

$$
\varphi_{\text {min }}^{F, S, l}(x)=\inf l \circ F(x)=\left\{\begin{array}{ll}
\infty & \text { if } x \in[-1,0) \\
l_{1}+l_{2}-\left(l_{1}+l_{2}\right) x & , \text { if } x \in[0,1]
\end{array},\right.
$$

and for the maximum value functions $\varphi_{\max }^{F, S, l}: S \rightarrow \overline{\mathbb{R}}$

$$
\varphi_{\max }^{F, S}(x)=\sup l \circ F(x)=\left\{\begin{array}{ll}
-\infty & , \text { if } x \in[-1,0) \\
l_{1}+l_{2} & , \text { if } x \in[0,1]
\end{array} .\right.
$$

Obviously, $\operatorname{dom}(F, S)=\operatorname{dom}(l \circ F, S)=[0,1]$ is convex and it is easy to see that $F \in \mathscr{C}_{u}\left(S, Y, \mathbb{R}_{+}^{2}\right)$ and $l \circ F \in \mathscr{C}_{u}\left(S, \mathbb{R}, \mathbb{R}_{+}\right)$holds for all $l \in C^{\star}=\mathbb{R}_{+}^{2}$. Moreover, for all $l \in C^{\star}=\mathbb{R}_{+}^{2}$ the minimum value functions $\varphi_{\min }^{F, S, l}$ are convex on $S=[-1,1]$ but the maximum value functions $\varphi_{\max }^{F, S, l}$ are convex only on $\operatorname{dom}(F, S)=\operatorname{dom}(l \circ F, S)=[0,1]$.

Note that in a real linear space $Y$ partially ordered by a convex cone $C \subset Y$ the equivalences

$$
A \preccurlyeq_{C}^{u} B \Leftrightarrow-B \preccurlyeq_{C}^{\ell}-A \Leftrightarrow B \preccurlyeq_{-C}^{\ell} A
$$

hold for $A, B \subset Y$ by definition of the $\ell$-less relation and the $u$-less relation. In contrast the concepts of $\ell$-type $C$-convexity and of $u$-type $C$-convexity of set-valued maps cannot be mutually converted by simple transformations. For set-valued maps only the following equivalences

$$
H \in \mathscr{C}_{u}(S, Y, C) \Leftrightarrow-H \in \mathscr{C}_{u}(S, Y,-C) \text { and } H \in \mathscr{C}_{\ell}(S, Y, C) \Leftrightarrow-H \in \mathscr{C}_{\ell}(S, Y,-C)
$$

are fulfilled. Thus Theorem 3.2 cannot be obtained directly from Theorem 3.1 at least by using such simple approaches. 


\section{QUASICONVEXITY OF SET-VALUED MAPS AND EXTREMAL VALUE FUNCTIONS}

There exist several concepts for quasiconvexity of set-valued maps. A systematization and an in-depth analysis of these concepts using the $\ell$-less relation and the $u$-less relation can be found in [7]. In this section we want to study the relations between quasiconvex set-valued maps and the quasiconvexity of their extremal value functions. Note that by Theorem 3.1 and Theorem 3.2 (under the additional assumptions formulated there) the convexity of a set-valued map is equivalent to the fact that the corresponding extremal value functions are convex for all $l \in C^{\star}$. In contrast, for none of the quasiconvexity concepts for set-valued maps (see the forthcoming Definition 4.1 and Definition 4.2) such an equivalent characterization by the quasiconvexity of the corresponding extremal value functions is known. In the following, we will give at least partial answers to the questions which quasiconvexity concepts are in both cases sufficient to guarantee the quasiconvexity of all these extremal value functions.

4.1. $\ell$-type quasiconvex set-valued maps. We start with the definition of the different quasiconvexity concepts based on the $\ell$-less relation.

Definition 4.1. [7, Definition 3.2] Let $X$ and $Y$ be real linear spaces, let $Y$ be partially ordered by a convex cone $C \subset Y$, let $S$ be a nonempty convex subset of $X$, and let $F: S \rightrightarrows Y$ be a set-valued map with $\operatorname{dom}(F, S) \neq \emptyset$. The map $F$ is called

(a) ( $\ell 1)$-type $C$-quasiconvex on $S$ if, for any convex subset $A$ of $Y$, the set

$$
\left\{x \in \operatorname{dom}(F, S) \mid F(x)+A \preccurlyeq_{C}^{\ell}\left\{0_{Y}\right\}\right\}
$$

is convex.

(b) ( $\ell 2)$-type $C$-quasiconvex on $S$ if, for any $y \in Y$, the set

$$
\left\{x \in \operatorname{dom}(F, S) \mid F(x) \preccurlyeq_{C}^{\ell}\{y\}\right\}
$$

is convex, which equivalently means that for any nonempty convex subset $A$ of $Y$ the set

$$
\left\{x \in \operatorname{dom}(F, S) \mid F(x) \preccurlyeq_{C}^{\ell} A\right\}
$$

is convex.

(c) ( $\ell 3)$-type $C$-quasiconvex on $S$ if, for any $x^{1}, x^{2} \in \operatorname{dom}(F, S)$ and $\lambda \in(0,1)$, it holds

$$
F\left(\lambda x^{1}+(1-\lambda) x^{2}\right) \preccurlyeq_{C}^{\ell}\left(F\left(x^{1}\right)+C\right) \cap\left(F\left(x^{2}\right)+C\right) .
$$

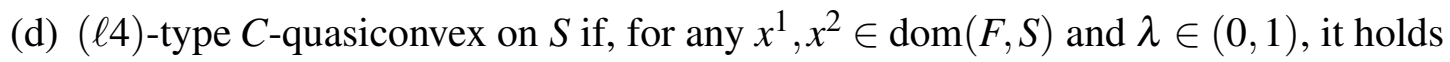

$$
F\left(\lambda x^{1}+(1-\lambda) x^{2}\right) \preccurlyeq_{C}^{\ell} F\left(x^{1}\right) \text { or } F\left(\lambda x^{1}+(1-\lambda) x^{2}\right) \preccurlyeq_{C}^{\ell} F\left(x^{2}\right) .
$$

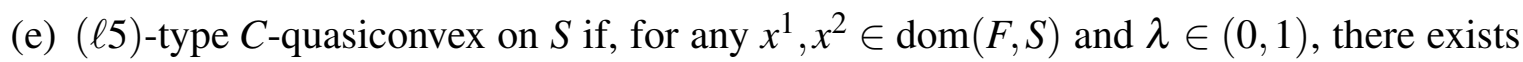
$t \in[0,1]$ such that

$$
F\left(\lambda x^{1}+(1-\lambda) x^{2}\right) \preccurlyeq_{C}^{\ell} t F\left(x^{1}\right)+(1-t) F\left(x^{2}\right) .
$$

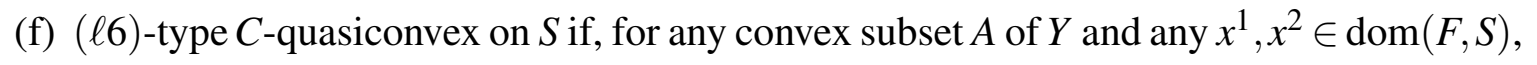

$$
t F\left(x^{1}\right)+(1-t) F\left(x^{2}\right)+A \preccurlyeq_{C}^{\ell}\left\{0_{Y}\right\} \text { for any } t \in[0,1]
$$

implies

$$
F\left(\lambda x^{1}+(1-\lambda) x^{2}\right)+A \preccurlyeq_{C}^{\ell}\left\{0_{Y}\right\} \text { for any } \lambda \in(0,1)
$$




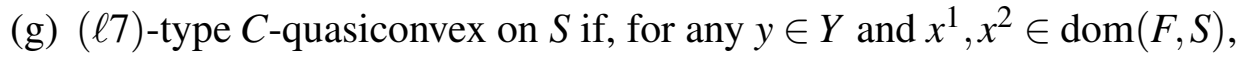

$$
t F\left(x^{1}\right)+(1-t) F\left(x^{2}\right) \preccurlyeq_{C}^{\ell}\{y\} \text { for any } t \in[0,1]
$$

implies

$$
F\left(\lambda x^{1}+(1-\lambda) x^{2}\right) \preccurlyeq_{C}^{\ell}\{y\} \text { for any } \lambda \in(0,1) .
$$

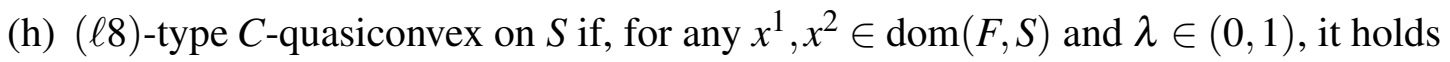

$$
F\left(\lambda x^{1}+(1-\lambda) x^{2}\right) \preccurlyeq_{C}^{\ell} \bigcap_{t \in[0,1]}\left(t F\left(x^{1}\right)+(1-t) F\left(x^{2}\right)+C\right) .
$$

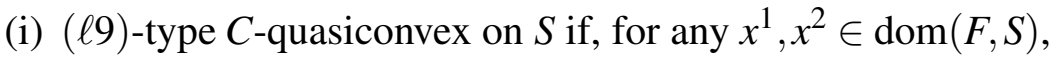

$$
F\left(x^{1}\right) \preccurlyeq_{C}^{\ell} F\left(x^{2}\right)
$$

implies

$$
F\left(\lambda x^{1}+(1-\lambda) x^{2}\right) \preccurlyeq_{C}^{\ell} F\left(x^{2}\right) \text { for any } \lambda \in(0,1) .
$$

Again, based on this and [7], we will denote by

$$
\mathscr{Q}_{\ell 1}(S, Y, C), \mathscr{Q}_{\ell 2}(S, Y, C), \ldots, \mathscr{Q}_{\ell 9}(S, Y, C)
$$

the classes of all $(\ell 1)$-type $C$-quasiconvex, $(\ell 2)$-type $C$-quasiconvex, $\ldots$, and $(\ell 9)$-type $C$ quasiconvex set-valued maps on $S$, respectively. Moreover, in [7, Proposition 3.4], it was proved that

$$
\begin{array}{ll} 
& \mathscr{Q}_{\ell 4}(S, Y, C) \\
\subsetneq & \mathscr{Q}_{\ell 5}(S, Y, C) \\
\subsetneq & \mathscr{Q}_{\ell 1}(S, Y, C)=\mathscr{Q}_{\ell 6}(S, Y, C) \\
\subsetneq & \mathscr{Q}_{\ell 2}(S, Y, C)=\mathscr{Q}_{\ell 3}(S, Y, C)=\mathscr{Q}_{\ell 7}(S, Y, C)=\mathscr{Q}_{\ell 8}(S, Y, C) \\
\subsetneq & \mathscr{Q}_{\ell 9}(S, Y, C),
\end{array}
$$

(see also [7, Example 3.5]) and according to [7, Example 4.1], it holds

$$
\mathscr{C}_{\ell}(S, Y, C) \not \subset \mathscr{Q}_{\ell 4}(S, Y, C) \text { and } \mathscr{C}_{\ell}(S, Y, C) \subset \mathscr{Q}_{\ell 5}(S, Y, C) \text {. }
$$

Note that the concept of ( $\ell 9)$-type $C$-quasiconvexity for set-valued maps defined above in Definition 4.1 can be interpreted as a generalization of the quasiconvexity (in the sense of Jahn) of vector-valued maps. If $f: S \rightarrow Y$ is again a vector-valued map and we define the set-valued map $F: S \rightrightarrows Y$ by $F(x):=\{f(x)\}$ for all $x \in S$, then $f$ is quasiconvex (in the sense of Jahn) on $S$ if and only if $F \in \mathscr{Q}_{\ell 9}(S, Y, C)$. As explained in much more detail in [7] all $C$-quasiconvexity concepts of Definition 4.1 are based on corresponding vector-valued counterparts (cf. [7, Definition 2.1, Remark 2 (a)] and references therein). For more background literature (some with different terminology) according to the various concepts we refer also for instance to $[6,10,11,12]$.

If additionally $C-C=Y$ is fulfilled (which is equivalent to the fact that $\left(\left\{y^{1}\right\}+C\right) \cap\left(\left\{y^{2}\right\}+\right.$ $C) \neq \emptyset$ holds for all $\left.y^{1}, y^{2} \in Y\right)$, then for all $F \in \mathscr{Q}_{\ell 2}(S, Y, C)=\mathscr{Q}_{\ell 3}(S, Y, C)=\mathscr{Q}_{\ell 7}(S, Y, C)=$ $\mathscr{Q}_{\ell 8}(S, Y, C)$ the domain $\operatorname{dom}(F, S)$ is a convex set (cf. [6]). In contrast to this, if $F \in \mathscr{Q}_{\ell 1}(S, Y, C)=$ $\mathscr{Q}_{\ell 6}(S, Y, C)$ and if we chose $A:=Y$ in (4.1), then we obtain by the definition of an ( $\left.\ell 1\right)$-type $C$-quasiconvex set-valued map on $S$ the convexity of the set $\left\{x \in \operatorname{dom}(F, S) \mid F(x)+Y \preccurlyeq_{C}^{\ell}\right.$ $\left.\left\{0_{Y}\right\}\right\}=\operatorname{dom}(F, S)$. Thus $\operatorname{dom}(F, S)$ is by using (4.2) convex for all $F \in \mathscr{Q}_{\diamond}(S, Y, C)$ with $\diamond \in\{\ell 1, \ell 4, \ell 5, \ell 6\}$. 
Moreover, under the assumptions of Theorem 3.1 it is shown in [11] that $F \in \mathscr{Q}_{\ell 2}(S, Y, C)=$ $\mathscr{Q}_{\ell 3}(S, Y, C)=\mathscr{Q}_{\ell 7}(S, Y, C)=\mathscr{Q}_{\ell 8}(S, Y, C)$ is fulfilled if the minimum value function $\varphi_{\text {min }}^{F, S, l}$ is quasiconvex on $S$ for all $l \in C^{\star}$. On the other hand the following lemma provides a sufficient condition for the quasiconvexity of the minimum value functions $\varphi_{\min }^{F, S, l}$ for all $l \in C^{\star}$.

Lemma 4.1. [6, Lemma 1.1 (A1)] Let $X$ be a real linear space, let $Y$ be a real topological linear space partially ordered by a convex cone $C \subset Y$, let $S$ be a nonempty convex subset of $X$, let $F: S \rightrightarrows Y$ be a set-valued map with $\operatorname{dom}(F, S) \neq \emptyset$, and let $l \in C^{\star}$. Then it holds:

If $l \circ F \in \mathscr{Q}_{\ell 2}\left(S, \mathbb{R}, \mathbb{R}_{+}\right)=\mathscr{Q}_{\ell 3}\left(S, \mathbb{R}, \mathbb{R}_{+}\right)=\mathscr{Q}_{\ell 7}\left(S, \mathbb{R}, \mathbb{R}_{+}\right)=\mathscr{Q}_{\ell 8}\left(S, \mathbb{R}, \mathbb{R}_{+}\right)$, then the minimum value function $\varphi_{\min }^{F, S, l}$ is quasiconvex on $S$.

Using Lemma 4.1, the following result for the extreme directions of the dual cone $C^{\star}$, denoted by $\operatorname{extd}\left(C^{\star}\right)$, can be proved. Recall that $l \in \operatorname{extd}\left(C^{\star}\right)$ if and only if $l \in C^{\star} \backslash\left\{0_{Y^{\star}}\right\}$ and $l=l_{1}+l_{2}$ with $l_{1}, l_{2} \in C^{\star}$ implies $l_{1}, l_{2} \in\{\alpha l \mid \alpha \geq 0\}$.

Proposition 4.1. [6, Proposition 3.1] Let $X$ be a real linear space, let $Y$ be Banach space partially ordered by a closed convex cone $C \subset Y$ with $C-C=Y$, and let $l \in \operatorname{extd}\left(C^{\star}\right)$. Then it holds:

If $F \in \mathscr{Q}_{\ell 2}(S, Y, C)=\mathscr{Q}_{\ell 3}(S, Y, C)=\mathscr{Q}_{\ell 7}(S, Y, C)=\mathscr{Q}_{\ell 8}(S, Y, C)$, then $l \circ F \in \mathscr{Q}_{\ell 2}\left(S, \mathbb{R}, \mathbb{R}_{+}\right)=$ $\mathscr{Q}_{\ell 3}\left(S, \mathbb{R}, \mathbb{R}_{+}\right)=\mathscr{Q}_{\ell 7}\left(S, \mathbb{R}, \mathbb{R}_{+}\right)=\mathscr{Q}_{\ell 8}\left(S, \mathbb{R}, \mathbb{R}_{+}\right)$and thus the minimum value function $\varphi_{\text {min }}^{F, S}$ is quasiconvex on $S$.

In [6, Theorem 3.1], under additional assumptions even an equivalent characterization of maps $F \in \mathscr{Q}_{\diamond}(S, Y, C)$ with $\diamond \in\{\ell 2, \ell 3, \ell 7, \ell 8\}$ via the quasiconvexity of the minimum value functions $\varphi_{\min }^{F, S, l}$ for all $l \in \operatorname{extd}\left(C^{\star}\right)$ is given. We will show in the following Theorem 4.1 that the quasiconvexity of the minimum value functions $\varphi_{\min }^{F, S, l}$ for all $l \in C^{\star}$ can be guaranteed if $F \in \mathscr{Q}_{\diamond}(S, Y, C)$ with $\diamond \in\{\ell 4, \ell 5\}$ holds. Therefore we need the following auxiliary result.

Lemma 4.2. Let $X$ be a real linear space, let $Y$ be a real topological linear space partially ordered by a convex cone $C \subset Y$, let $S$ be a nonempty convex subset of $X$, and let $F: S \rightrightarrows Y$ be a set-valued map with $\operatorname{dom}(F, S) \neq \emptyset$. If $F \in \mathscr{Q}_{\ell 5}(S, Y, C)$, then it holds $l \circ F \in \mathscr{Q}_{\ell 5}\left(S, \mathbb{R}, \mathbb{R}_{+}\right)$for all $l \in C^{\star}$.

Proof. Let $F \in \mathscr{Q}_{\ell 5}(S, Y, C), l \in C^{\star}$, and $H:=l \circ F$. Then it follows $\operatorname{dom}(F, S)=\operatorname{dom}(H, S)$ by definition and thus the convexity of $\operatorname{dom}(H, S)$. Moreover, let $x^{1}, x^{2} \in \operatorname{dom}(F, S)=\operatorname{dom}(H, S)$ and $\lambda \in(0,1)$. Then by definition there exists $t \in[0,1]$ such that

$$
F\left(\lambda x^{1}+(1-\lambda) x^{2}\right) \preccurlyeq_{C}^{\ell} t F\left(x^{1}\right)+(1-t) F\left(x^{2}\right) .
$$

Moreover, let $\bar{\alpha} \in t H\left(x^{1}\right)+(1-t) H\left(x^{2}\right)$. Then there exist $y^{1} \in F\left(x^{1}\right)$ and $y^{2} \in F\left(x^{2}\right)$ such that

$$
\bar{\alpha}=t l\left(y^{1}\right)+(1-t) l\left(y^{2}\right)=l\left(t y^{1}+(1-t) y^{2}\right),
$$

and

$$
t y^{1}+(1-t) y^{2} \in t F\left(x^{1}\right)+(1-t) F\left(x^{2}\right) \subset F\left(\lambda x^{1}+(1-\lambda) x^{2}\right)+C .
$$

Thus there exist $\bar{y} \in F\left(\lambda x^{1}+(1-\lambda) x^{2}\right)$ and $k \in C$ such that

$$
t y^{1}+(1-t) y^{2}=\bar{y}+k \text {. }
$$

Since $l \in C^{\star}$, it holds

$$
l(\bar{y})=l\left(t y^{1}+(1-t) y^{2}-k\right)=l\left(t y^{1}+(1-t) y^{2}\right)-l(k) \leq l\left(t y^{1}+(1-t) y^{2}\right)=\bar{\alpha},
$$


and we obtain

$$
\bar{\alpha} \in\{l(\bar{y})\}+\mathbb{R}_{+} \subset l\left(F\left(\lambda x^{1}+(1-\lambda) x^{2}\right)\right)+\mathbb{R}_{+}=H\left(\lambda x^{1}+(1-\lambda) x^{2}\right)+\mathbb{R}_{+} .
$$

Hence, it follows

$$
\begin{aligned}
& t H\left(x^{1}\right)+(1-t) H\left(x^{2}\right) \subset H\left(\lambda x^{1}+(1-\lambda) x^{2}\right)+\mathbb{R}_{+} \\
\Leftrightarrow & H\left(\lambda x^{1}+(1-\lambda) x^{2}\right) \preccurlyeq \mathbb{R}_{+}^{\ell} t H\left(x^{1}\right)+(1-t) H\left(x^{2}\right),
\end{aligned}
$$

and thus $H=l \circ F \in \mathscr{Q}_{\ell 5}\left(S, \mathbb{R}, \mathbb{R}_{+}\right)$.

As a direct consequence of Lemma 4.1, Lemma 4.2, and (4.2), we obtain the announced result.

Theorem 4.1. Let $X$ be a real linear space, let $Y$ be a real topological linear space partially ordered by a convex cone $C \subset Y$, let $S$ be a nonempty convex subset of $X$, and let $F: S \rightrightarrows Y$ be a set-valued map with $\operatorname{dom}(F, S) \neq \emptyset$. If $F \in \mathscr{Q}_{\diamond}(S, Y, C)$ with $\diamond \in\{\ell 4, \ell 5\}$, then the minimum value function $\varphi_{\min }^{F, S, l}$ is quasiconvex on $S$ for all $l \in C^{\star}$.

4.2. $u$-type quasiconvex set-valued maps. In this subsection, we show with the forthcoming Theorem 4.2 a result similar to Theorem 4.1 for $u$-type quasiconvex set-valued maps, i.e., we study the question, which quasiconvexity concept based on the $u$-less relation guarantees the quasiconvexity for all maximum value functions $\varphi_{\max }^{F, S, l}$ with $l \in C^{\star}$. We start again with the definition of the different concepts.

Definition 4.2. [7, Definition 3.3] Let $X$ and $Y$ be real linear spaces, let $Y$ be partially ordered by a convex cone $C \subset Y$, let $S$ be a nonempty convex subset of $X$, and let $F: S \rightrightarrows Y$ be a set-valued map with $\operatorname{dom}(F, S) \neq \emptyset$. The map $F$ is called

(a) (u1)-type $C$-quasiconvex on $S$ if, for any convex subset $A$ of $Y$, the set

$$
\left\{x \in \operatorname{dom}(F, S) \mid F(x) \preccurlyeq_{C}^{u} A\right\}
$$

is convex.

(b) (u2)-type $C$-quasiconvex on $S$ if, for any $y \in Y$, the set

$$
\left\{x \in \operatorname{dom}(F, S) \mid F(x) \preccurlyeq_{C}^{u}\{y\}\right\}
$$

is convex.

(c) (u3)-type $C$-quasiconvex on $S$ if, for any $x^{1}, x^{2} \in \operatorname{dom}(F, S)$ and $\lambda \in(0,1)$, it holds

$$
F\left(\lambda x^{1}+(1-\lambda) x^{2}\right) \preccurlyeq_{C}^{u} F\left(x^{1}\right) \cup F\left(x^{2}\right) .
$$

(d) (u4)-type $C$-quasiconvex on $S$ if, for any $x^{1}, x^{2} \in \operatorname{dom}(F, S)$ and $\lambda \in(0,1)$, it holds

$$
F\left(\lambda x^{1}+(1-\lambda) x^{2}\right) \preccurlyeq_{C}^{u} F\left(x^{1}\right) \text { or } F\left(\lambda x^{1}+(1-\lambda) x^{2}\right) \preccurlyeq_{C}^{u} F\left(x^{2}\right) .
$$

(e) (u5)-type $C$-quasiconvex on $S$ if, for any $x^{1}, x^{2} \in \operatorname{dom}(F, S)$ and $\lambda \in(0,1)$, there exists $t \in[0,1]$ such that

$$
F\left(\lambda x^{1}+(1-\lambda) x^{2}\right) \preccurlyeq_{C}^{u} t F\left(x^{1}\right)+(1-t) F\left(x^{2}\right) .
$$

(f) (u6)-type $C$-quasiconvex on $S$ if, for any nonempty convex subset $A$ of $Y$ and any $x^{1}, x^{2} \in$ $\operatorname{dom}(F, S)$,

$$
t F\left(x^{1}\right)+(1-t) F\left(x^{2}\right) \preccurlyeq_{C}^{u} A \text { for any } t \in[0,1]
$$


implies

$$
F\left(\lambda x^{1}+(1-\lambda) x^{2}\right) \preccurlyeq_{C}^{u} A \text { for any } \lambda \in(0,1) .
$$

(g) (u7)-type $C$-quasiconvex on $S$ if, for any $y \in Y$ and $x^{1}, x^{2} \in \operatorname{dom}(F, S)$,

$$
t F\left(x^{1}\right)+(1-t) F\left(x^{2}\right) \preccurlyeq_{C}^{u}\{y\} \text { for any } t \in[0,1]
$$

implies

$$
F\left(\lambda x^{1}+(1-\lambda) x^{2}\right) \preccurlyeq_{C}^{u}\{y\} \text { for any } \lambda \in(0,1) .
$$

(h) (u8)-type $C$-quasiconvex on $S$ if, for any $x^{1}, x^{2} \in \operatorname{dom}(F, S)$ and $\lambda \in(0,1)$, it holds

$$
F\left(\lambda x^{1}+(1-\lambda) x^{2}\right) \preccurlyeq_{C}^{u} \bigcup_{t \in[0,1]}\left(t F\left(x^{1}\right)+(1-t) F\left(x^{2}\right)\right) .
$$

(i) (u9)-type $C$-quasiconvex on $S$ if, for any $x^{1}, x^{2} \in \operatorname{dom}(F, S)$,

$$
F\left(x^{1}\right) \preccurlyeq_{C}^{u} F\left(x^{2}\right)
$$

implies

$$
F\left(\lambda x^{1}+(1-\lambda) x^{2}\right) \preccurlyeq_{C}^{u} F\left(x^{2}\right) \text { for any } \lambda \in(0,1) .
$$

Again based on this and again according to [7], we will denote in the following by

$$
\mathscr{Q}_{u 1}(S, Y, C), \mathscr{Q}_{u 2}(S, Y, C), \ldots, \mathscr{Q}_{u 9}(S, Y, C)
$$

the classes of all $(u 1)$-type $C$-quasiconvex, $(u 2)$-type $C$-quasiconvex, $\ldots$, and $(u 9)$-type $C$ quasiconvex set-valued maps on $S$, respectively. According to [7, Proposition 3.6], it holds

$$
\begin{array}{ll} 
& \mathscr{Q}_{u 4}(S, Y, C) \\
\subset & \mathscr{Q}_{u 3}(S, Y, C) \cap \mathscr{Q}_{u 5}(S, Y, C) \\
\subset & \mathscr{Q}_{u 3}(S, Y, C) \cup \mathscr{Q}_{u 5}(S, Y, C) \\
\subset & \mathscr{Q}_{u 8}(S, Y, C) \\
\subset & \mathscr{Q}_{u 1}(S, Y, C)=\mathscr{Q}_{u 6}(S, Y, C) \\
\subset & \mathscr{Q}_{u 2}(S, Y, C)=\mathscr{Q}_{u 7}(S, Y, C) .
\end{array}
$$

Additionally, if $F(x)-C$ is convex for all $x \in \operatorname{dom}(F, S)$, then

$$
\mathscr{Q}_{u 1}(S, Y, C)=\mathscr{Q}_{u 6}(S, Y, C) \subset \mathscr{Q}_{u 8}(S, Y, C) \cap \mathscr{Q}_{u 9}(S, Y, C) .
$$

Moreover, (see [7, Example 3.7 and Example 4.1]), we have

$$
\begin{aligned}
& \mathscr{C}_{u}(S, Y, C) \not \subset \mathscr{Q}_{u 3}(S, Y, C), \mathscr{C}_{u}(S, Y, C) \subset \mathscr{Q}_{u 5}(S, Y, C), \\
& \mathscr{Q}_{u 3}(S, Y, C) \not \subset \mathscr{Q}_{u 4}(S, Y, C), \mathscr{Q}_{u 5}(S, Y, C) \not \subset \mathscr{Q}_{u 4}(S, Y, C), \\
& \mathscr{Q}_{u 8}(S, Y, C) \not \subset \mathscr{Q}_{u 3}(S, Y, C), \mathscr{Q}_{u 8}(S, Y, C) \not \subset \mathscr{Q}_{u 5}(S, Y, C), \\
& \mathscr{Q}_{u 2}(S, Y, C)=\mathscr{Q}_{u 7}(S, Y, C) \not \subset \mathscr{Q}_{u 1}(S, Y, C)=\mathscr{Q}_{u 6}(S, Y, C), \\
& \mathscr{Q}_{u 9}(S, Y, C) \not \subset \mathscr{Q}_{u 1}(S, Y, C)=\mathscr{Q}_{u 6}(S, Y, C), \\
& \mathscr{Q}_{u 2}(S, Y, C)=\mathscr{Q}_{u 7}(S, Y, C) \not \subset \mathscr{Q}_{u 9}(S, Y, C), \text { and } \\
& \mathscr{Q}_{u 9}(S, Y, C) \not \subset \mathscr{Q}_{u 2}(S, Y, C)=\mathscr{Q}_{u 7}(S, Y, C) .
\end{aligned}
$$

Clearly, the concept of $(u 9)$-type $C$-quasiconvexity for set-valued maps defined above can in analogy to the ( $\ell 9)$-type $C$-quasiconvexity be interpreted as a generalization of the quasiconvexity (in the sense of Jahn) of vector-valued maps. If again $f: S \rightarrow Y$ is a vector-valued map and the set-valued map $F: S \rightrightarrows Y$ is defined by $F(x):=\{f(x)\}$ for all $x \in S$, then $f$ is quasiconvex (in the sense of Jahn) on $S$ if and only if $F \in \mathscr{Q}_{u 9}(S, Y, C)$, and $F \in \mathscr{Q}_{\ell 9}(S, Y, C)$ if and 
only if $F \in \mathscr{Q}_{u 9}(S, Y, C)$ (in addition to this we refer to [7, Remark 2 (f)]). Similarly to the concepts of $C$-quasiconvexity based on the $\ell$-less relation all $C$-quasiconvexity concepts defined in Definition 4.2 are again based on corresponding vector-valued counterparts (cf. [7, Definition 2.1, Remark 2 (b)]). Note that the notions of (u2)-type, (u3)-type, (u4)-type, (u5)-type, and (u8)-type $C$-quasiconvexity were introduced in [9].

For the proof of the announced theorem, we need the following two auxiliary results.

Lemma 4.3. Let $X$ be a real linear space, let $Y$ be a real topological linear space partially ordered by a convex cone $C \subset Y$, let $S$ be a nonempty convex subset of $X$, let $F: S \rightrightarrows Y$ be a set-valued map with $\operatorname{dom}(F, S)=S$, and let $l \in C^{\star}$. Then it holds:

If $l \circ F \in \mathscr{Q}_{u 5}\left(S, \mathbb{R}, \mathbb{R}_{+}\right)$, then the maximum value function $\varphi_{\max }^{F, S, l}$ is quasiconvex on $S$.

Proof. Let $l \in C^{\star}, l \circ F \in \mathscr{Q}_{u 5}\left(S, \mathbb{R}, \mathbb{R}_{+}\right)$, and $H:=l \circ F$. In order to prove that the corresponding maximum value function $\tilde{\varphi}:=\varphi_{\max }^{F, S, l}$ is convex on $S$, it suffices by Lemma 2.2 to show that its strict level set

$$
\tilde{\varphi}_{<}^{-1}(S, \alpha):=\left\{x \in S \mid \varphi_{\max }^{F, S, l}(x)<\alpha\right\}
$$

is a convex set for all $\alpha \in \mathbb{R}$. Let therefore $\alpha \in \mathbb{R}$ be arbitrarily chosen. If $\tilde{\varphi}_{<}^{-1}(S, \alpha)=\emptyset$, then nothing is to prove. Therefore, let $x^{1}, x^{2} \in \tilde{\varphi}_{<}^{-1}(S, \alpha)$ and $\lambda \in[0,1]$. Then it holds $x^{1}, x^{2} \in S=$ $\operatorname{dom}(F, S)=\operatorname{dom}(H, S),-\infty<\varphi_{\max }^{F, S, l}\left(x^{1}\right)<\alpha$, and $-\infty<\varphi_{\max }^{F, S, l}\left(x^{2}\right)<\alpha$. Using the convexity of $S=\operatorname{dom}(F, S)=\operatorname{dom}(H, S)$, we obtain

$$
\lambda x^{1}+(1-\lambda) x^{2} \in S=\operatorname{dom}(F, S)=\operatorname{dom}(H, S) .
$$

We have to show that

$$
\varphi_{\max }^{F, S, l}\left(\lambda x^{1}+(1-\lambda) x^{2}\right) \leq \alpha
$$

holds. If $\lambda \in\{0,1\}$, then (4.4) is obviously fulfilled. Hence, we may assume $\lambda \in(0,1)$. Since $H \in \mathscr{Q}_{u 5}\left(S, \mathbb{R}, \mathbb{R}_{+}\right)$, there exists $t \in[0,1]$ such that

$$
H\left(\lambda x^{1}+(1-\lambda) x^{2}\right) \subset t H\left(x^{1}\right)+(1-t) H\left(x^{2}\right)-\mathbb{R}_{+} .
$$

Now, let $\beta \in \mathbb{R}$ be chosen such that

$$
\max \left\{\varphi_{\max }^{F, S, l}\left(x^{1}\right), \varphi_{\max }^{F, S, l}\left(x^{2}\right)\right\}<\beta<\alpha .
$$

Then it holds

$$
t H\left(x^{1}\right) \subset\{t \beta\}-\mathbb{R}_{+} \text {and }(1-t) H\left(x^{2}\right) \subset\{(1-t) \beta\}-\mathbb{R}_{+},
$$

and we obtain

$$
\begin{aligned}
H\left(\lambda x^{1}+(1-\lambda) x^{2}\right) & \subset t H\left(x^{1}\right)+(1-t) H\left(x^{2}\right)-\mathbb{R}_{+} \\
& \subset\left(\{t \beta\}-\mathbb{R}_{+}\right)+\left(\{(1-t) \beta\}-\mathbb{R}_{+}\right)-\mathbb{R}_{+} \\
& =\{\beta\}-\mathbb{R}_{+} .
\end{aligned}
$$

It follows that

$$
\varphi_{\max }^{F, S, l}\left(\lambda x^{1}+(1-\lambda) x^{2}\right)=\sup H\left(\lambda x^{1}+(1-\lambda) x^{2}\right) \leq \beta<\alpha .
$$

This completes the proof.

Lemma 4.4. Let $X$ be a real linear space, let $Y$ be a real topological linear space partially ordered by a convex cone $C \subset Y$, let $S$ be a nonempty convex subset of $X$, and let $F: S \rightrightarrows Y$ be a set-valued map with $\operatorname{dom}(F, S) \neq \emptyset$. If $F \in \mathscr{Q}_{u 5}(S, Y, C)$, then it holds $l \circ F \in \mathscr{Q}_{u 5}\left(S, \mathbb{R}, \mathbb{R}_{+}\right)$ for all $l \in C^{\star}$. 
Proof. Let $F \in \mathscr{Q}_{u 5}(S, Y, C), l \in C^{\star}, H:=l \circ F, \lambda \in(0,1)$, and $x^{1}, x^{2} \in \operatorname{dom}(F, S)=\operatorname{dom}(H, S)$. Then there exists $t \in[0,1]$ such that

$$
F\left(\lambda x^{1}+(1-\lambda) x^{2}\right) \subset t F\left(x^{1}\right)+(1-t) F\left(x^{2}\right)-C \neq \emptyset .
$$

Obviously, it is sufficient to show that

$$
H\left(\lambda x^{1}+(1-\lambda) x^{2}\right) \preccurlyeq \mathbb{R}_{+}^{\ell} t H\left(x^{1}\right)+(1-t) H\left(x^{2}\right)
$$

holds.

If $\lambda x^{1}+(1-\lambda) x^{2} \notin \operatorname{dom}(F, S)=\operatorname{dom}(H, S)$, then (4.6) is trivially fulfilled by using (2.2). Hence, we may assume $\lambda x^{1}+(1-\lambda) x^{2} \in \operatorname{dom}(F, S)=\operatorname{dom}(H, S)$ and let

$$
\bar{\alpha} \in H\left(\lambda x^{1}+(1-\lambda) x^{2}\right) \text {. }
$$

Then there exists $\bar{y} \in F\left(\lambda x^{1}+(1-\lambda) x^{2}\right)$ such that $\bar{\alpha}=l(\bar{y})$. From (4.5), we see that there must exist $y^{1} \in F\left(x^{1}\right), y^{2} \in F\left(x^{2}\right)$, and $k \in C$ such that $\bar{y}=t y^{1}+(1-t) y^{2}-k$. Hence, we obtain by $l \in C^{\star}$ that

$$
\bar{\alpha}=l(\bar{y})=l\left(t y^{1}+(1-t) y^{2}-k\right)=t l\left(y^{1}\right)+(1-t) l\left(y^{2}\right)-l(k) \leq t l\left(y^{1}\right)+(1-t) l\left(y^{2}\right),
$$

and consequently

$$
\begin{aligned}
\bar{\alpha} & \in t\left\{l\left(y^{1}\right)\right\}+(1-t)\left\{l\left(y^{2}\right)\right\}-\mathbb{R}_{+} \\
& \subset t l\left(F\left(x^{1}\right)\right)+(1-t) l\left(F\left(x^{2}\right)\right)-\mathbb{R}_{+} \\
& =t H\left(x^{1}\right)+(1-t) H\left(x^{2}\right)-\mathbb{R}_{+} .
\end{aligned}
$$

Hence, it follows again

$$
\Leftrightarrow \begin{aligned}
& H\left(\lambda x^{1}+(1-\lambda) x^{2}\right) \subset t H\left(x^{1}\right)+(1-t) H\left(x^{2}\right)-\mathbb{R}_{+} \\
\Leftrightarrow \quad & H\left(\lambda x^{1}+(1-\lambda) x^{2}\right) \preccurlyeq_{\mathbb{R}_{+}}^{u} t H\left(x^{1}\right)+(1-t) H\left(x^{2}\right),
\end{aligned}
$$

which implies that $H=l \circ F \in \mathscr{Q}_{u 5}\left(S, \mathbb{R}, \mathbb{R}_{+}\right)$. This completes the proof.

As a direct consequence of Lemma 4.3, Lemma 4.4, and (4.3) we obtain here the following result:

Theorem 4.2. Let $X$ be a real linear space, let $Y$ be a real topological linear space partially ordered by a convex cone $C \subset Y$, let $S$ be a nonempty convex subset of $X$, and let $F: S \rightrightarrows Y$ be a set-valued map with $\operatorname{dom}(F, S)=S$. If $F \in \mathscr{Q}_{\diamond}(S, Y, C)$ with $\diamond \in\{u 4, u 5\}$, then the maximum value function $\varphi_{\max }^{F, S, l}$ is quasiconvex on $S$ for all $l \in C^{\star}$.

\section{CONCLUSIONS}

A known result of Benoist and Popovici [6, Theorem 2.1] provides that a set-valued map $F: S \rightrightarrows Y$ is $\ell$-type $C$-convex on $S$ if and only if the scalar-valued minimum value function $\varphi_{\text {min }}^{F, S, l}$ is convex on $S$ for all $l \in C^{\star}$. With the new Theorem 3.2, a similar result regarding the equivalent characterization of $u$-type $C$-convex set-valued maps is formulated. We prove that a set-valued map $F: S \rightrightarrows Y$ is $u$-type $C$-convex on $S$ if and only if the scalar-valued maximum value function $\varphi_{\max }^{F, S, l}$ is convex on $S$ for all $l \in C^{\star}$. Moreover, for quasiconvex set-valued maps based on the $\ell$-less relation (see [7, Definition 3.2]) and based on the $u$-less relation (see [7, Definition 3.3]), it is shown that the minimum value function $\varphi_{\min }^{F, S, l}$ is quasiconvex on $S$ for all $l \in C^{\star}$ provided that $F$ is ( $\left.\ell 5\right)$-type $C$-quasiconvex on $S$ (see Theorem 4.1 ) and that the maximum value function $\varphi_{\max }^{F, S, l}$ is quasiconvex on $S$ for all $l \in C^{\star}$ provided that $F$ is (u5)-type 
$C$-quasiconvex on $S$ (see Theorem 4.2). Using the hierarchical orders (4.2) and (4.3), it should be clarified in a next step if this is true also for $(\ell 1) /(\ell 6)$-type $C$-quasiconvex maps and for (u8)-type $C$-quasiconvex maps, respectively.

\section{Acknowledgments}

The authors thank the two anonymous referees for their careful reading and their very helpful comments on the first version of this manuscript. The work of the second author was funded by the Deutsche Forschungsgemeinschaft (DFG, German Research Foundation) - Project-ID 392195690.

\section{REFERENCES}

[1] A.A. Khan, C. Tammer, C. Zălinescu. Set-Valued Optimization - An Introduction with Applications, Springer-Verlag, Berlin, Heidelberg, 2015.

[2] D. Kuroiwa, Existence Theorems of Set Optimization with Set-Valued Maps. Manuscript Shimane University, Japan, 1997.

[3] D. Kuroiwa, Natural Criteria of Set-Valued Optimization, Manuscript Shimane University, Japan, 1998.

[4] D. Kuroiwa, Some duality theorems of set-valued optimization with natural criteria, In: W. Takahashi, T. Tanaka, (ed.) Proceedings of the International Conference on Nonlinear Analysis and Convex Analysis, in Niigata, Japan, pp. 221-228, World Scientific, Singapore, 1999.

[5] J. Jahn, Vectorization in set optimization, J. Optim. Theory Appl. 167 (2015), 783-795.

[6] J. Benoist, N. Popovici. Characterizations of convex and quasiconvex set-valued maps, Math. Meth. Oper. Res. 57 (2003), 427-435.

[7] K. Seto, D. Kuroiwa, N. Popovici, A systematization of convexity and quasiconvexity concepts for set-valued maps, defined by l-type and u-type preorder relations, Optimization, 67 (2018), 1077-1094.

[8] J. Jahn. Vector Optimization - Theory, Applications, and Extensions, Springer-Verlag, Berlin, Heidelberg, 2004.

[9] D. Kuroiwa, Convexity for Set-Valued Maps and Optimization, PhD thesis, Niigata: Niigata University, 1996.

[10] G.P. Crespi, D. Kuroiwa, M. Rocca, Quasiconvexity of set-valued maps assures well-posedness of robust vector optimization, Ann. Oper. Res. 251 (2017), 89-104.

[11] D. Kuroiwa, Convexity for set-valued maps. Appl. Math. Lett. 9 (1996), 97-101.

[12] D.T. Luc, C. Vargas, A saddlepoint theorem for set-valued maps, Nonlinear Anal. 18 (1992), 1-7. 